\title{
EVACUATION ALGORITHM OF ARMOURED VEHICLES
}

\author{
Ivan MINEVSKI, Yordan HRISTOV, Ilian LILOV \\ "Vasil Levski" National Military University, Veliko Tarnovo, Bulgaria \\ ivan_minevski@abv.bg, danchohr@abv.bg, inl@abv.bg
}

\begin{abstract}
This publication analyses the main stages of evacuation of stuck and mind-bound armoured vehicles. A methodology and an algorithm have been developed for towing vehicles in cases of insufficient traction power of the traction engine.
\end{abstract}

\section{Keywords: evacuation, armoured vehicles}

\section{Introduction}

While operating the armoured vehicles pass very rough ground where they are at risk of getting stuck into marshy land, getting stranded into pits, ditches or riverbeds with muddy sediments, and overturning and sinking in water plains. Recovery of damaged machines and repairing them greatly depends on timely evacuation.

Evacuation of the vehicles is a comprehensive methodology for organizing and realizing tow of stuck and sunk machines and their transportation to the places for mending or the places for further evacuation. It is carried out in accordance with the circumstances at hand, the location, the type of sinking or stranding, and the technical repair of the vehicles.

The main factors that affect the timely execution of evacuation are the following:

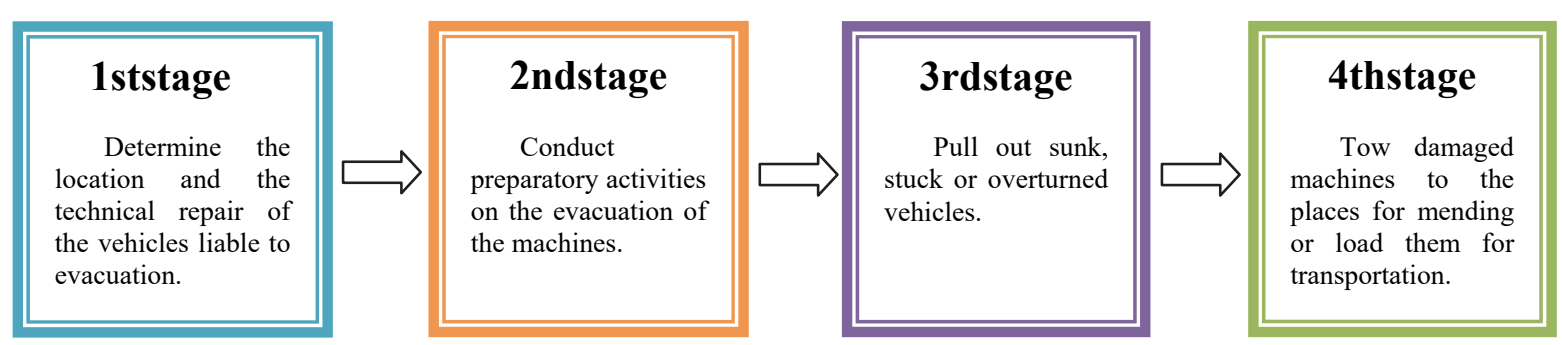

Figure 1: Stages of organizing and conducting machine evacuation 
$\mathbf{1}^{\text {st }}$ stage: Determine the location and the technical condition of the vehicles liable to evacuation.

When determining the location and the condition of the vehicles we specify:

$\checkmark$ The presence and condition of the crew, the brand and classification of the vehicle;

$\checkmark$ The type of damage (stranding) of the machine and the necessary means for its evacuation and mending;

$\checkmark$ The contamination of the vehicle and the location with radioactive or poisonous substances;

$\checkmark$ The presence of minefields, fences or other obstacles impending the evacuation.

The necessary data concerning sunk machines are supplemented with the help of frogmen and the information about the location of the vehicle is added to the one concerning the depth of the body of water, the type of its bed, the temperature of the water, the speed of the water current, etc.

$2^{\text {nd }}$ stage: Conduct preparatory activities on the evacuation of the machines.

Depending on the certain circumstances available, the preparatory activities on the evacuation of the machines consists of:

$\checkmark$ Tracing, demining and clearing roads for approach, maneuvering and evacuation;

$\checkmark$ Controlling the level of contamination of the machines liable to evacuation;

$\checkmark \quad$ Making rigging and other supplementary gear;

$\checkmark$ Clearing the area around the machine; making exits, flooring and anchors (if necessary);

$\checkmark$ Replacing or removing the damaged centres and units hindering the evacuation of the machines.

3rdstage: Pull out sunk, stuck or overturned vehicles.

Before pulling out the vehicles it is necessary to tighten all evacuation ropes which connect the tow-truck, the elements of the polyspast (if used) and the machine evacuated. This activity is required in order to check the correct building of the polyspast, the condition of all evacuation ropes and their reliable connection.
When pulling the machines all safety regulation must be observed. In addition to that all parties in the towing - i.e. the chief executive officer, the tow-truck driver and the driver of the evacuated machine (if any) - must maintain visual contact.

$4^{\text {th }}$ stage: Tow damaged machines to the places for mending or load them for transportation.

Transportation of towed machines to the places for mending or loading them for further evacuation is done in the following ways:

$\checkmark$ Under their own power, on condition that the machines have working engines, transmission, suspension and steering systems;

$\checkmark$ Under tow, on condition that the vehicles have working suspension system;

$\checkmark$ Loaded on special carriers used for transporting heavy vehicles.

When organizing and carrying out evacuation of vital importance it is necessary to define the tension force which will operate on the stuck, or sunk machine while towing. In order to estimate the necessary tension force (effort) (P) we must analyze the following factors:

$\checkmark$ Type and technical repair of the vehicle;

$\checkmark$ Type of stranding, depth of sinking;

$\checkmark \quad$ The condition of the soil and the bed of the body of water;

$\checkmark$ The characteristics of the location, declivity degree of the terrain and its accessibility;

$\checkmark \quad$ The number of preparatory activities.

In order to diminish the number of activities and the time for organizing and conducting the evacuation it is necessary to compare the tension force $(\mathrm{P})$ with the mass of the stuck, or sunk machine (M).

According to the ratio between the necessary tension force (effort) $(\mathrm{P})$ and the mass of the machine (M) strandings (sinkings) fall into the following categories. [1]

Low level of stranding (sinking) is the one which requires a few preparatory activities before towing the machine and 
the necessary tension force does not exceed the mass of the evacuated machine.

$P=M$

For example: stranded machine in a ditch with gradual slopes; sunk up to the middle of the roadwheels in a marshy location, etc.

$>$ Medium level of stranding (sinking) is the one which requires a few preparatory activities before towing the machine and the necessary tension force exceeds the mass of the evacuated machine with no more than 1.5 times.

$P=1,5 M$

For example: stranded machine in a ditch with steep slopes; sunk up to the top of the roadwheels in a marshy location; overturned machine in a ditch, etc.

$>$ High level of stranding (sinkintiong) is the one which requires a considerable number of preparatory activities before towing the vehicle and the necessary tension force exceeds its mass up to three times.

$p=3 M$

For example: stranded vehicle sunk to the cupola in a marshy location; a machine sunk in a body of water with a stony bed and gradual banks.

Extreme level of stranding (sinking) is the one which requires a considerable number of preparatory activities before towing the machine and the necessary tension force exceeds its mass with more than three times.

$P>3 M$

For example: fully sunk machine in a marshy location; overturned vehicle in a body of water, etc.

When estimating the traction force for towing a vehicle it is necessary to take into account the technical repair of the evacuated machine which is a factor with a great impact on the development of the evacuation process.

\section{Polyspasts, function and types.}

It is a common incidence when organizing evacuation to calculate the tractive effort and end up with a necessary effort for evacuating a stranded vehicle several times lower than the power produced by the towtruck Q. In order to increase the power (of the tow-truck) to the necessary power it is advisable to use a system of pulleys called polyspast (block and tackle).[2] The polyspast (Figure 2) is a combination of fixed and moveable pulleys connected with a rope which can increase the force of the tow-truck.

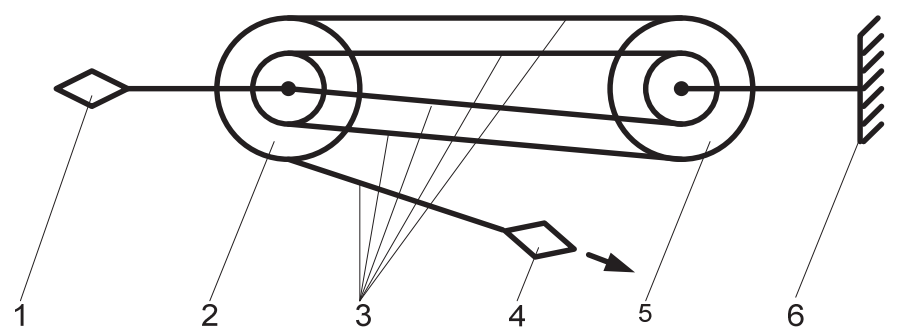

Figure 2: Diagram of a polyspast: 1-evacuated vehicle; 2-movable pulley; 3-rope sections; 4tow-truck; 5-fixed pulley; 6-fixed mounting point (anchor)

The basic parts of a polyspast are:

$\checkmark$ Movable pulley - connected to the evacuated vehicle;

$\checkmark$ Fixed pulley - connected to the anchor;

$\checkmark$ Evacuation rope - consisting of steel wires in cross laid strands; $\checkmark$ Anchor - used for mounting the fixed pulley on the spot. Natural objects at the location (i.e. a tree, a stump, a rock) can be used as anchors as well as objects especially made for the evacuation operation like equipment and machinery with the necessary mass. 
The ideal mechanical advantage of the polyspast (i) indicates the times the towtruck traction force is increased. It is calculated with the formula:

$i=\frac{P}{q}$

where: $\mathrm{P}$ - necessary traction force for towing;

Q - available traction force (of the towtruck).

Polyspasts are simple and compound.

All the pulleys in the simple polyspast are threaded with a single rope (Figure 3 ).

If the end of the rope where the pulling force is applied appears from the movable pulley (Figure 3a), the ideal mechanical advantage is calculated with the formula (6). On condition that the end of the rope comes out of the fixed pulley (Figure $3 \mathrm{~b}$ ),

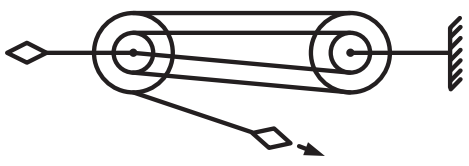

a.

Figure 3: Diagram of building a simple polyspast machine.

$i=n=m+1$

$l-m-n-1$ polyspast;

$\mathrm{m}$ - number of pulleys. has to pass.

b.

the ideal mechanical advantage is calculated with the formula (7). The latter type of polyspast is used when the local conditions require directing the pulling force in the direction of the evacuated

where: $\mathrm{n}$ - number of rope sections of the

When placing the elements of the simple polyspast on terrain it is important to estimate the distance between the two pulleys correctly; that distance must be longer than the one the evacuated vehicle

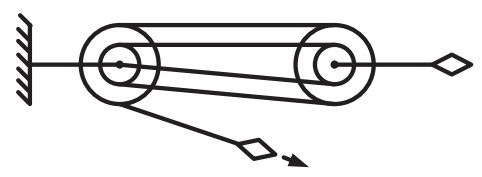

The length of the rope for building a simple polyspast $\mathrm{L}$ is estimated with the following formula:

$L=5 \cdot n+a, m$

where:

$\mathrm{S}$ - the distance the evacuated machine has to pass;

$\mathrm{n}$ - the number of rope sections of the polyspast;

$a$ - additional length of the rope having in mind the slacks and winds round the pulleys $(a=10 \div 15 \mathrm{~m}$.)

Before building the polyspast it is necessary to clear a road to be used by the tow-truck. The distance the truck passes $\mathrm{S}_{1}$ is calculated with the following formula:

$S_{1}=S_{i} i, \mathrm{~m}$

where:

$\mathrm{S}$ - the distance the evacuated machine has to pass;

$\mathrm{i}$ - the mechanical advantage of the polyspast.
The maximum mechanical advantage when using a simple polyspast consisting of two double pulleys is 5 .

When a bigger mechanical advantage is needed it is necessary to build a compound polyspast which consists of two or more simple polyspasts connected consecutively (Figure 4). The mechanical advantage of a compound polyspast $i_{h}$ is equal to the product of the mechanical advantages of all simple polyspasts comprising it.

$i_{h}=i_{1}, i_{2} i_{n}$

The length of the rope $L_{n}$ of each simple polyspast included in the structure of the compound polyspast is calculated by multiplying: the road the evacuated machine has to pass $\mathrm{S}$; the number of the rope sections of the current polyspast $n_{n}$; the product of the rope sections of the preceding polyspasts $\left(\mathrm{n}_{\mathrm{p} 1}, \mathrm{n}_{\mathrm{p} 2}, \ldots \ldots\right)$.

$L_{n}-S \cdot r_{n} \cdot T_{p 1} \cdot r_{p 2} \cdots$ 


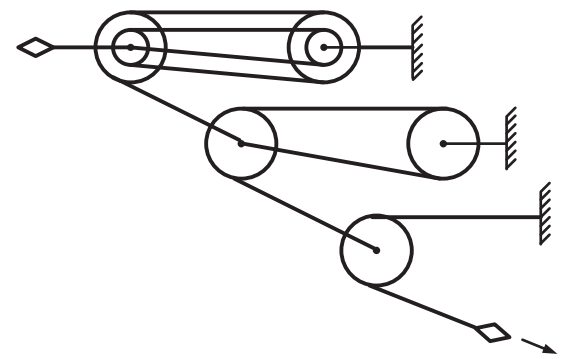

Figure 4: Diagram of a compound polyspast
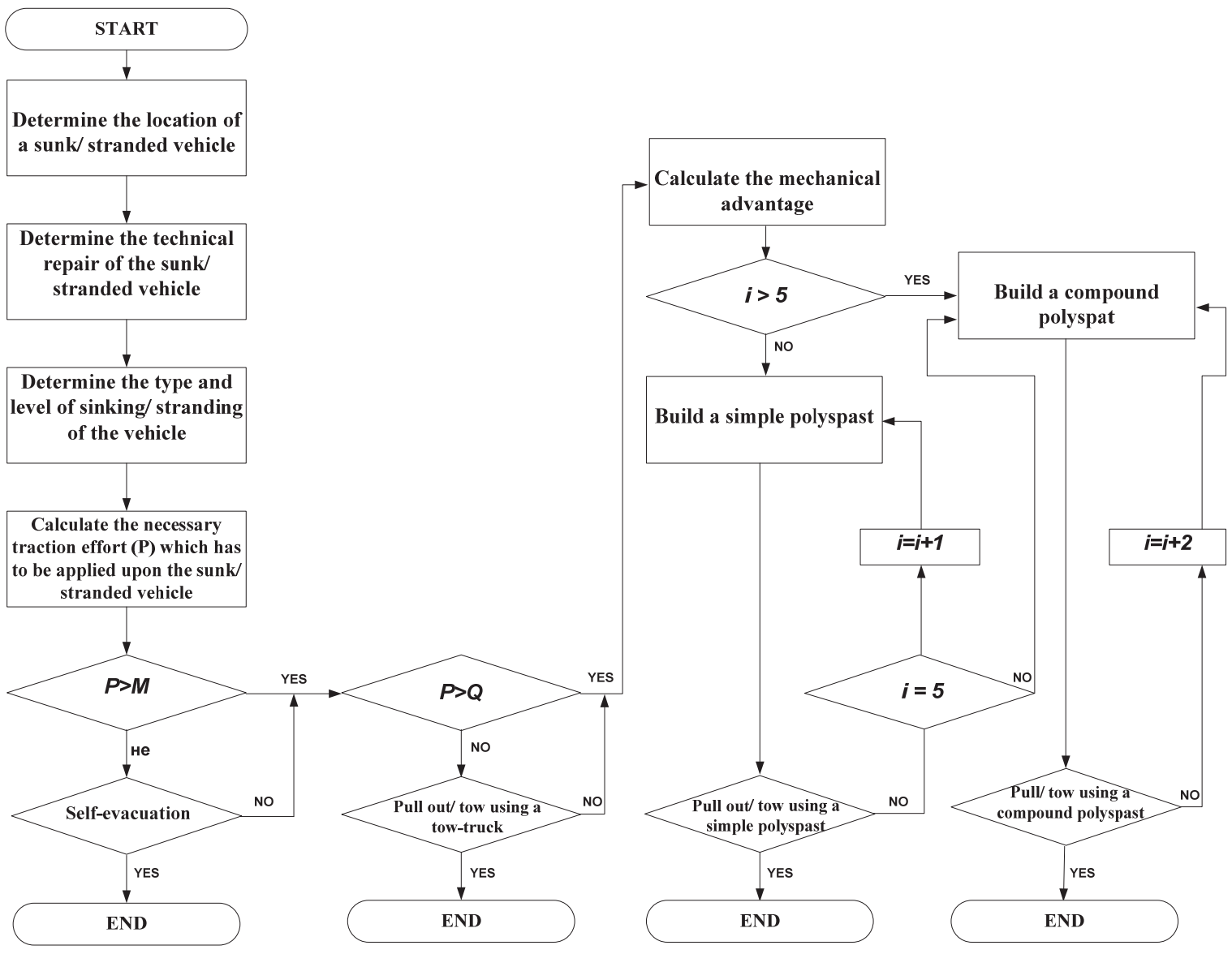

Figure 5: Algorithm for evacuation of stranded (sunk) vehicles

\section{Algorithm for evacuation of stranded (sunk) vehicles.}

The algorithm for evacuation of stranded (sunk) vehicles Figure 5. has been developed on the basis of analyzing the basic activities in the stages of evacuation. This algorithm consists of sequence of regulated actions and calculations which are followed by actual practical operations. In case of a failure at any of the stages the algorithm gives corrective operations which lead to the desired end, namely evacuation of the stuck machine. After calculating the necessary traction effort the vehicle is moved out under its own power. If selfevacuation is impossible, the algorithm proposes towing by a tow-truck or calculating and building a simple polyspast according to the available and the necessary traction force/ effort. In accordance with the calculated mechanical advantage and the 
result of the towing the algorithm proposes building a compound polyspast and possible corrective operations.

\section{Conclusions}

The proposed algorithm encompasses all possible variants of means for evacuation of stranded vehicles. It gives the opportunity to take the optimal decision on the method of towing a sunk (stranded) machine having in mind all circumstances concerning the location, the technical repair of the machine, the level of sinking and the available technical means and forces for evacuation. The algorithm has been put into practice during trainings and drills with military students.

This algorithm finds application when organizing and carrying out evacuation of military maintenance equipment as well as with the specialized search and rescue forces.

\section{References}

[1] Dragolov, D. Evacuation of military maintenance equipment. Sofia.1997

[2] Minevski, I. Risk analysis of the evacuation transport vehiles. Scientific conference „Fire and emergence 2007” Sofia 2007 